\title{
Thermal Management and Concurrent System Design of a Wearable Multicomputer
}

\author{
Cristina H. Amon, Senior Member, IEEE, Eric R. Egan, Asim Smailagic, Associate Member, IEEE, \\ and Daniel P. Siewiorek, Fellow, IEEE
}

\begin{abstract}
This paper describes the concurrent system design and thermal management of the Navigator 2 which is used as a computerized maintenance manual for aircraft inspection with speech recognition capabilities. The Navigator 2 is a wearable computer that includes a novel dual architecture, spread spectrum radio, and variable gain amplifier (VGA) head-mounted display. The semi-custom electronic design includes two electronic boards-a custom-designed system board and a 486-based processor board. The system board captures glue logic functions and provides support for two PCMCIA slots, a power management microcontroller, memory backup batteries, and a power supply.

The thermal design of the Navigator 2 develops concurrently with the overall design in a series of stages. A framework of concurrent thermal engineering consisting of three basic stages is used to maintain interdisciplinary interaction while satisfying thermal design goals.

In the first stage of the thermal design, a cooling arrangement that meets the needs of other disciplines is proposed, and an enhanced-conduction thermal design with aluminum heat spreaders and active power-saving is explored.

In the second stage, the thermal contact between heat spreaders and electronic components is optimized, and physical experimentation is performed with liquid heat sinks and conductive elastomers as thermal contact interfaces.

In the third stage, numerical simulations are performed to ascertain the effectiveness of the thermal design, giving the thermal designer flexibility to change critical parameters and perform sensitivity analyses.

A simplified computational model is used to investigate the performance of thermal interface devices and the effect of the heat spreader design on the maximum electronic component temperatures. Although the simplified model proves adequate for thermal design purposes, a detailed geometrically-accurate computational model assesses the adequacy of the exposed heat spreader surface area and predicts temperature distributions with better agreement to the experimental measurements on the Navigator2.
\end{abstract}

Index Terms - Concurrent thermal design, portable electronics, thermal management, wearable computers.

\section{INTRODUCTION}

W EARABLE computers are robust portable computers that allow information to be delivered at any place and any time. They are designed to be dependable, comfortably

Manuscript received June 1996; revised January 9, 1997. This paper was presented at the ITHERM Conference, Orlando, FL, May 30-June 1, 1996. This work was supported by the Engineering Design Research Center, National Science Foundation Grants CTS-9 630 801, MIP-9403473, and DMI-9415001, and Advanced Research Project Agency Contract DABT 63-95-C-0026.

The authors are with Carnegie Mellon University, Pittsburgh, PA 15213 USA (e-mail: amon@cmu.edu).

Publisher Item Identifie S 1070-9886(97)03428-8.

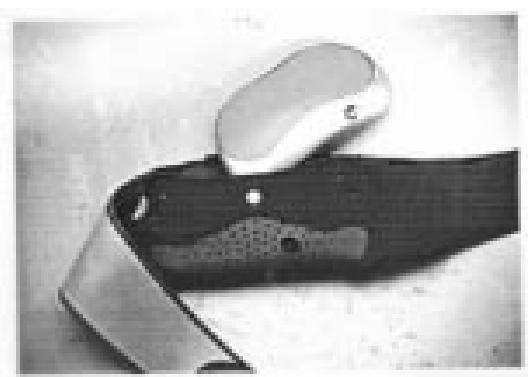

(a)

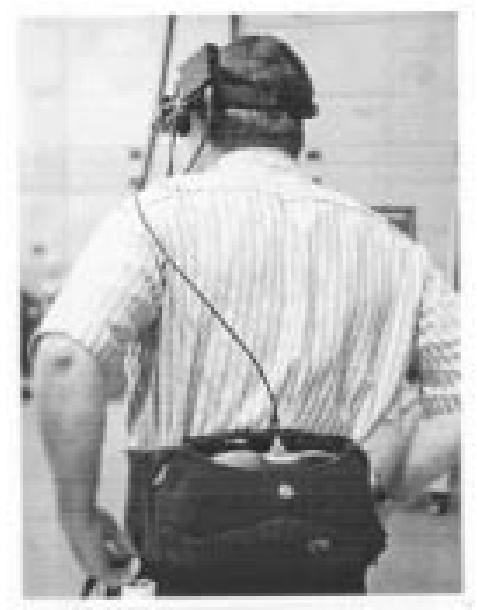

(b)

Fig. 1. Photographs of Navigator2: (a) with its wearable harness and (b) belt-worn.

worn, and highly functional. The goal of the thermal design for any computer is to determine an efficien means of cooling the computer's electronic components such that the operating temperature of each component does not exceed manufacturersuggested limits for reliable operation. To meet this goal, the power and size of the computer, as well as its environment, must be taken into account to achieve an efficien thermal design. Our research focuses on the thermal and system design of computers that are portable, lightweight, and rugged. These computers are meant to be worn and used on the body and are known as wearable computers [1], [14].

The main subject of discussion for this paper is the concurrent thermal design of the Navigator2 wearable computer. Photographs of the Navigator 2 with its wearable harness are shown in Fig. 1(a) and (b). The Navigator2 is the latest wearable computer designed and developed in the Wearable 
Computer Course during the spring semester of 1995 at Carnegie Mellon [2]. The Navigator2 is designed for Boeing aircraft inspection applications as a computerized maintenance manual. Some of its capabilities include global position sensing (GPS), speech recognition, wireless local area networks (LAN) communication, and variable gain amplifie (VGA) head-mounted display.

In addition to the thermal aspects of wearable computer design, it is necessary to concurrently satisfy other design criteria. A thermal designer cannot arbitrarily select the thermal design for electronic cooling. Industrial design considerations such as weight, size, and ergonomics of the wearable computer affect, for example, the size, shape, and placement of heat spreaders that conduct heat away from the heat-generating electronics. Furthermore, power consumption and placement of the heat-producing components are typically a result of decisions made by the electronics team. This type of design procedure involves many different areas of specialization from electronics to thermal design to industrial design, requiring good interdisciplinary design skills and cooperation [8]. As the different phases of design from other disciplines mature, the thermal design must adjust accordingly. Concurrent design at each stage of the process is the only way to assure that the thermal design meets the requirements of the changing wearable computer. Concurrent thermal design involves the use of numerical simulations and analytical approximations to predict at each stage the thermal limitations of the design [8]. Therefore, concurrent design must keep pace with all of the developments in the overall design of the wearable computer.

The modern designer should consider the many choices available in the selection of the appropriate devices used in the thermal design [6]. In most desk-top computer designs, forced air cooling has been employed [3], however, the unique constraints of wearable computers renders air ventilation slots on the outercase unfeasible and the use of fans impractical. Wearable computers are designed to be small, lightweight, and ergonomic. They must often operate in wet and dirty environments for applications such as aircraft maintenance [15]. Hence, removal of heat simply by conduction with plate heat sinks and heat spreaders is preferred since it is economical, consumes no power, and needs no moving parts. Therefore, our objective in the thermal design of wearable computers is to optimize the conduction of heat from the electronic components to the outer casing as well as its removal by natural convection.

However, not all portable computers of this size rely solely on heat spreaders in their thermal management. Other devices such as thermoelectric modules or heat sinks with built-in fans can be used to cool the heat-producing components but require power to operate, thus inhibiting the battery life of the wearable computer. However, passive devices ranging from fla plate heat sinks and liquid heat sinks to miniature heat pipes can be employed without the need for power. Heat pipes are expensive, highly technological devices that offer excellent cooling ability, but we try to avoid their use unless especially high heat flu densities exist in the system. Many laptop computers rely on air ventilation as an inexpensive mode of cooling [13]. Other portable computer designers focus on the electronics, optimizing designs for low power consumption and high efficiency For example, high heat-producing voltage regulators can usually be conditioned to be much more powerefficien [5]. In another case, a well known maker of computers created a separate company to design new processors for its palmtop computer partially based on the need for low power dissipation. It further created software with interrupts, creating an event-driven system to minimize power consumption [4]. In an attempt to effectively manage the heat produced by future laptops with processors that consume up to $20 \mathrm{~W}$, another company plans to route the heat to the keyboard. With so many various strategies to adequately cool portable computers, the simplicity of a heat spreader can be appreciated as an effective solution to the thermal design.

\section{Electronics OF the NAVIGATOR2}

Navigator2 is the sixth generation of CMU wearable computers and uses a semi-custom electronic design, consisting of two printed circuit boards with 29 chips. The Navigator2 electronics includes two major electronic boards: a system board and a processor board (Epson's CARDIO-486). The custom-designed system board captures all glue logic functions and also provides support for two PCMCIA slots of types 2 and 3, power management using a PIC16C71 microcontroller, memory backup lithium batteries, and power supply. The type 2 PCMCIA card slot accommodates a TERI speech recognition card, while the type 3 PCMCIA slot is used for an Integral Peripherals $260 \mathrm{MB}$ hard disk. The main board and processor card are connected via an ISA bus.

The major design considerations taken into account when evaluating the processor/motherboard subsystem are:

1) modular hardware architecture supporting customization of input/output devices;

2) specifi constraints to satisfy the following wearable computer attributes: size, weight, form of input/output, thermal management, and battery consumption.

A technology survey phase identifie three major candidates for the processor board: Epson's CARDIO-486, Teknor's VIPer 803, and Ampro's CoreModule/486-II. The Epson card has superior dimensions of $85.6 \times 54 \times 5.5 \mathrm{~mm}$ and a typical $3.1 \mathrm{~W}$ power consumption. Also, it has VGA display support, $12 \mathrm{MB}$ on-board dynamic random access memory (DRAM), and a $33 \mathrm{Mhz}$ Intel $486 \mathrm{SL}$ processor. As for comparison, VIPer's dimensions are $122 \times 181 \times 10 \mathrm{~mm}$ and power consumption is $9.75 \mathrm{~W}$. Ampro's Coremodule dimensions are $90 \times 96 \times 23 \mathrm{~mm}$, and it has a power consumption of $4.65 \mathrm{~W}$.

An important design decision was to decouple main processing from speech processing. The TERI speech recognition card, with a $13 \mathrm{MHz}$ digital signal processor, is utilized for speech processing and renders a satisfactory speech recognition response time of less than $1 \mathrm{~s}$ per word with a 150 word vocabulary.

In the thermal analysis of the Navigator2, only the larger electronic components that generate a significan amount of heat are included in the numerical simulations. Table I provides a list of the components used in the numerical simulations along with the physical dimensions, thermal properties, and 
TABLE I

Main Heat-Producing Electronic Components of Navigator2

\begin{tabular}{|c|c|c|}
\hline Component & $\begin{array}{c}\text { Estimated } \\
\text { Power Level } \\
\text { (W) }\end{array}$ & $\begin{array}{c}\text { Maximum } \\
\text { Operating } \\
\text { Temperature } \\
\left({ }^{\circ} \mathrm{C}\right)\end{array}$ \\
\hline $\begin{array}{l}\text { Epson IO-486 Card-Type PC } \\
\text { (with 3.3V } 33 \mathrm{MHz} 486 \mathrm{SL} \text { Processor) }\end{array}$ & $4.5^{*}$ & 55 \\
\hline $\begin{array}{l}\text { 5V Voltage Regulator } \\
\text { (Power Trends 78ST305HC) }\end{array}$ & 1.5 & 60 \\
\hline $\begin{array}{l}\text { 3.3V Voltage Regulator } \\
\text { (Power Trends 78ST133HC) }\end{array}$ & 0.75 & 60 \\
\hline $\begin{array}{l}160 \text { MB Hard Disk } \\
\text { (Entegral Peripherals, Inc.) }\end{array}$ & 0.85 & 60 \\
\hline Total Operating Conditions & 7.60 & 55 \\
\hline
\end{tabular}

power ratings. The electronics of the Navigator 2 are positioned on both sides of one printed circuit board (PCB). The Epson Card, a compact electronic, aluminum-cased package containing the microprocessor and its necessary accessories, produces the largest amount of heat. It is in thermal contact with an aluminum heat spreader underneath the PCB that conducts the heat to the outer aluminum subframe. The aluminum heat spreader is between the aluminum subframe and bottom plastic housing of Fig. 2. The voltage regulators are directly in contact with the aluminum subframe, while the hard drive is not in thermal contact with the heat spreader or aluminum subframe.

\section{Power-SaVing Featues for the NAVIGATOR2 WEARABLE COMPUTER}

The electronic, software, and thermal design teams made it a priority to reduce the power consumption of the Navigator2 so that we could maximize battery life and minimize operating temperatures and thermal management requirements.

Power management for the Navigator2 has been done both at the hardware and software levels. At the hardware level, a programmable microcontroller, PIC16C71, performs power management functions. When powered up, the PIC initializes and registers its input/output (I/O) ports and then goes into SLEEP mode. It remains in this power-conserving mode until it is awakened by an INT interrupt. The INT interrupt is triggered when the user turns ON the Navigator2. The PIC turns on the green LED and then proceeds to do an analogto-digital (A/D) conversion on the main battery. If the battery is low, the green LED blinks, power is not turned onto the system, and the PIC goes back to SLEEP mode.

When the battery voltage is adequate, the PIC tests the lithium battery. In case the lithium voltage is low, the red LED blinks, and the power is not turned on, otherwise, the PIC initializes its TMR0 interrupt, turns on the power to the rest of the system, and enters the main loop of operation. The following functions have also been implemented: Current Overload, Main Battery Low, and Main Battery Warning messages. The PIC has the ability to receive these messages and act upon their receipt.

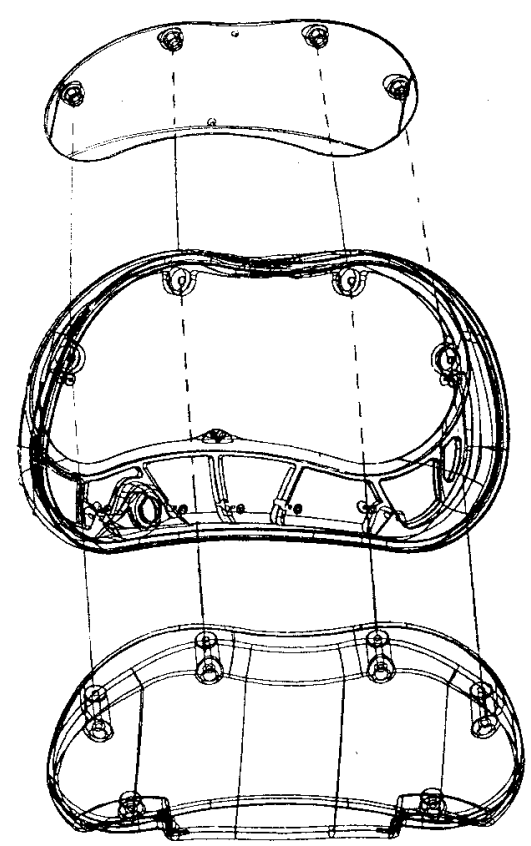

Fig. 2. Solid model showing assembly of Navigator2 housing.

In addition to the above power-saving features, the Epson processor card provides the Minimizer BIOS module with four main power-saving modes and different settings to control wake up and sleep modes. The power saving modes are: CPU Standby, Local Standby, Global Standby, and Suspend. CPU Standby checks the amount of time the CPU has been idle and after a period of time, in increments of $4 \mathrm{~s}$, stops the CPU clock and reduces power consumption. Local Standby is similar to the CPU Standby but detects the amount of time that a device has been idle and then turns off the power to that device. Global Standby makes the entire system enter global standby mode, and devices may be turned back into normal mode by an interrupt. The whole system is switched into the suspended mode after being idle for a certain period of time (also in increments of $4 \mathrm{~s}$ ). The system is relinquished from the suspended mode by a suspend button signal or by a realtime clock alarm. The Minimizer helps to significantl reduce power consumption in an idle system and in an active system with idle devices.

The CPU Standby mode can be set as low as $4 \mathrm{~s}$ or up to $17 \mathrm{~min}$. The Global Standby mode occurs only after the system has been in CPU Standby mode for a period of time between $4 \mathrm{~s}$ and $36 \mathrm{~h}$. In this mode, almost all devices go into the Standby state, further saving power consumption. The Auto Suspend state occurs only after a certain period of time has elapsed in Global Standby mode. The timer can be set from $4 \mathrm{~s}$ to $36 \mathrm{~h}$, and in this state the power is shut off for almost all devices. The recommended time switches for all of the settings given by the Navigator2 power management team are listed in Table II.

With the power management team reducing the overall power consumption and the thermal design team creating efficien conduction paths to dissipate the heat, the goal to obtain reliable operating temperatures for the components can be met. 
TABLE II

Software Power-Saving Features of Navigator2

\begin{tabular}{ll}
\multicolumn{1}{c}{ Mode } & \multicolumn{1}{c}{ Timer } \\
\hline CPU Standby & 4 seconds \\
Global Standby & 15 seconds \\
Auto Suspend & 5 minutes \\
Video & Disable \\
Serial 1 & 4 seconds \\
Serial 2 & 4 seconds \\
Hard Disk & 1 minute \\
TERI Card & 4 seconds \\
\hline
\end{tabular}

\section{Concurrent Design of Wearable Computers}

Due to the short design cycle required to compete successfully in today's global economy, the design of different disciplines involved in wearable computers must progress concurrently. Frequent communication and interaction is necessary to ensure that the overall design of the product matures efficiently allowing the interdisciplinary design process to accurately address all of the constraints of the various disciplines. The Navigator 2 design process can be described as a truly interdisciplinary concurrent design, where the disciplines involved are: electronics, mechanical engineering, industrial design, software, and human computer interaction.

Fig. 3 illustrates the design process and dependencies among these groups. The tasks are represented as bubbles. By following the tasks involving several disciplines, we can track down design dependencies as the design matures. For example, the task representing the choice of microphone, speech card, electronics, and software support for speech input and recognition can be accomplished only if all involved groups complete their contributions. This diagram can also be utilized as a beneficia tracking and monitoring tool for the overall design process.

As our concurrent design methodology produces rapidly prototyped wearable computer systems, the role of human factors is important in all stages of the design process, concluding with the design evaluation. Therefore, a separate column in Fig. 3 reflect the human computer interaction activities.

The Navigator2 housing fabrication involves molding and machining. The housing design follows an evolutionary path from initial drawings and mock-ups to a refine three-dimensional (3-D) solid model and stereolithography (SLA) model. Upon receiving the housing parts from the manufacturer, necessary post-production processes are applied where individual parts are finishe by spraying EMI shielding, adding color and/or texture, anodizing or coating aluminum surfaces, or silk-screening graphics onto the parts.

\section{A. Concurrent Thermal Engineering}

For wearable computers, thermal design cannot be an afterthought. Many disciplines are required to work in parallel with each contributing to the overall product: the wearable computer [16]. Communication with other disciplines is necessary to produce the most advantageous thermal design that satisfie not only thermal constraints but also the needs of other disciplines involved as well. For example, the electronic design team must be informed from the outset that high power electronics, in addition to inhibiting battery life, constitute

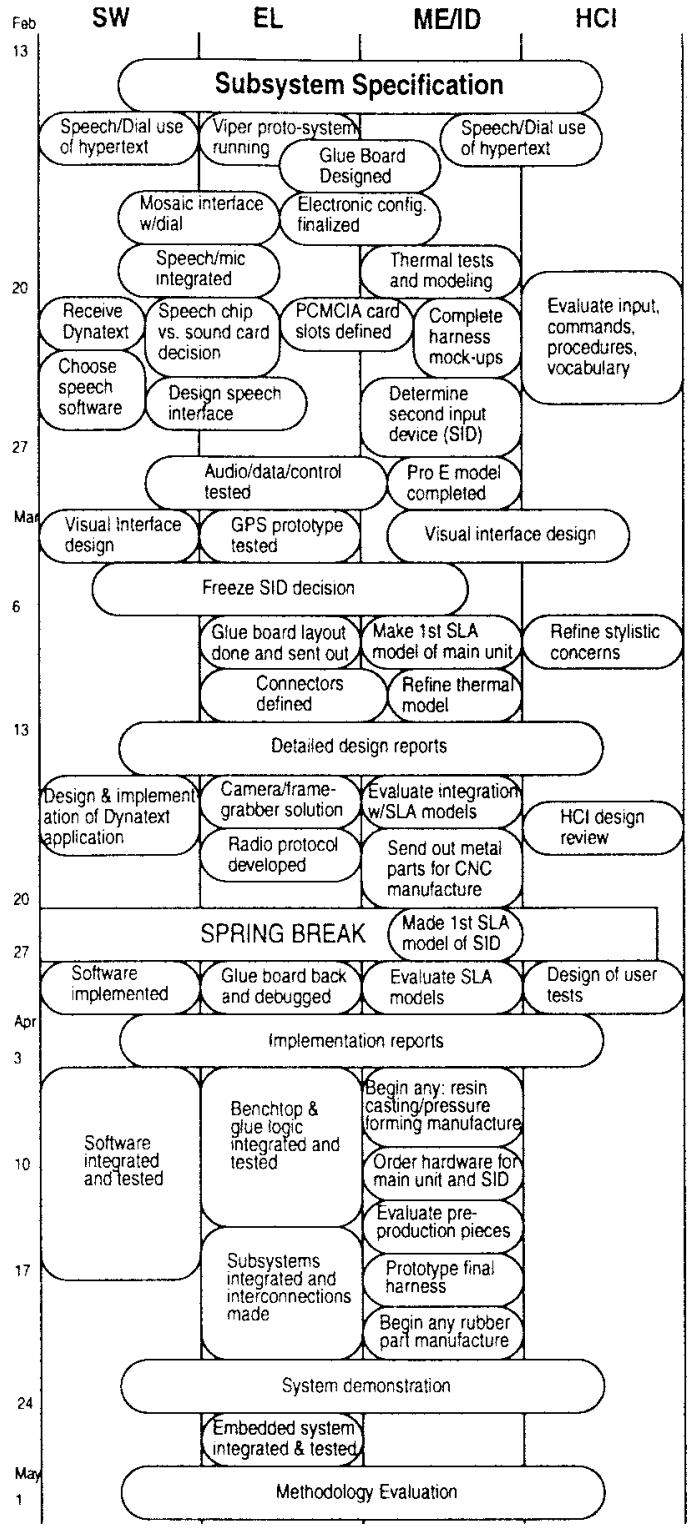

Fig. 3. Task dependency graph for concurrent design of Navigator2 depicting from left to right: software, electronic, mechanical, industrial, and human computer interaction interfaces.

an important thermal design issue which may impose component placement constraints. Also, industrial design issues must be addressed concurrently. For wearable computers, the outer casing of the housing must incorporate the heat sink to facilitate natural convection heat removal. The heat sink must not only satisfy thermal design but also aesthetic and ergonomic criteria and serve the dual purpose of dissipating heat and providing robustness and rigidity to the housing structure [1]. Because thermal design is highly dependent on and constrained by other disciplines, personal interaction with designers from other disciplines is necessary, thus demanding a cohesive design framework in which progress at all levels is performed concurrently.

Communication, however, is only one aspect of concurrent engineering. The thermal design must affect the design of the overall product at each stage of the design process thereby 
placing much responsibility on the thermal designer. As the design progresses, the thermal designer must employ those tools which are appropriate, such as analytical formulations, numerical simulations, and physical experimentation, to insure an adequate thermal solution.

\section{B. Framework of Concurrent Thermal Engineering}

With the application of wearable computers, the thermal design must be flexibl and responsive to changes in the designs from other disciplines. However, a systematic method using concurrent design principles [12] guides the thermal designer through a design process which consists of three stages for the Navigator 2 wearable computer. The firs stage of the thermal design is to select the type of cooling arrangement that is needed based on the specification of the electronics, preliminary layout, and power-level.

Then, iteration within this stage is necessary with analytical estimations to determine the appropriate passive cooling, conduction-enhancement method such that the main constraints are satisfied

In the second stage, the actual device(s) and the expected implementation are tested. The thermal management devices considered for the Navigator 2 are aluminum heat spreaders and thermal interface devices. For testing these devices, a physical experiment is constructed to validate their application for the wearable computer. With increased knowledge from the second stage, the thermal management device(s) can be selected to fulfil the demands of the previous stage. Then, the third stage begins which consists mainly of numerical simulations. In the numerical simulations, a conduction-only model of the wearable computer is usually adequate to obtain a rough approximation of the temperature distribution throughout its domain. For a more accurate prediction of the temperature distribution, a conjugate conduction/convection simulation is made before the fina prototype is constructed [1]. The conjugate conduction/convection simulation solves the energy equation for both the flui and solid domains. This simulation takes a substantial amount of time even with high speed, high capacity, computational facilities and is, therefore, used only in the last stage of the thermal design.

\section{Application of Concurrent Thermal Engineering to the Navigator2}

Because the Navigator2 is designed with a completely closed housing for rugged, wet, and dirty operating conditions, it is decided early in the design process that heat generated by the electronic components must be removed by enhanced conduction to the outer casing and then dissipated by natural convection. One of the focal points of the thermal design is the thermal contact between the electronics and the aluminum heat spreader since the air gap between the electronics and heat spreader constitutes the largest thermal resistance to be minimized.

Two thermal interface devices are tested with a heat sink test bed: a liquid heat sink (LHS) and a conductive elastomer. The heat sink test bed consists of eight 7805 voltage regulators which can produce between 3 and $20 \mathrm{~W}$ of power and is

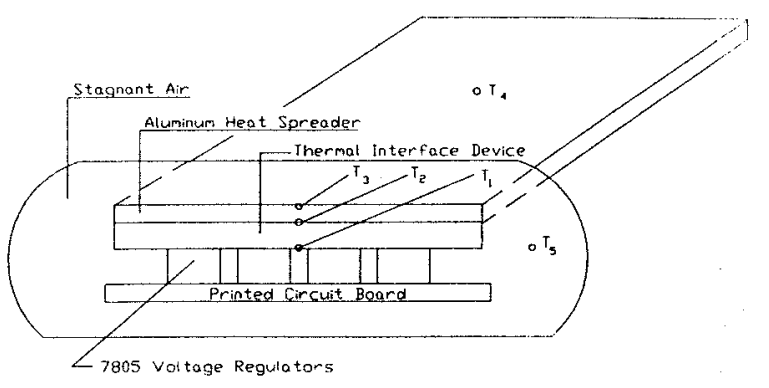

Fig. 4. Schematic showing thermocouple locations of heat sink test bed.

TABLE III

Heat Spreaders Used in Heat Sink Test Bed Experiments

\begin{tabular}{ccc}
\hline Designation & $\begin{array}{c}\text { Thickness } \\
\text { (in) }\end{array}$ & $\begin{array}{c}\text { Area Exposed to } \\
\text { Natural Air } \\
\text { Convection }\left(\text { in }^{2}\right)\end{array}$ \\
\hline A & $1 / 48$ & 36 \\
B & $1 / 16$ & 36 \\
C & $1 / 16$ & 108 \\
D & $1 / 8$ & 108 \\
\hline
\end{tabular}

depicted in Fig. 4. In these experiments, a 0.125 inch layer of the conductive elastomer out-performed the LHS by some $25 \%$, based on the overall temperature rise from the voltage regulators to the surrounding air inside the test bed. Also, the LHS posed implementation difficultie due to the necessity of even pressure distribution along the LHS packet. Thus, we chose the conductive elastomer as the thermal interface between the electronics and the heat spreader.

The third stage of thermal development consists of numerical simulations conducted to obtain a detailed prediction of the temperatures expected throughout the Navigator2. First, a simplifie rectangular geometry having similar dimensions to the actual wearable computer is used. For this computational model, the stagnant air inside the housing is modeled as a solid with the properties of air [11] and is later replaced by conservative convective boundary conditions. The second computational model of the Navigator2 is much more detailed, bearing a marked resemblance to the actual artifact. The stagnant air inside the housing is modeled with convective boundary conditions. Both models employ conduction-only simulations using boundary conditions to account for the flui mechanical effects of convection heat transfer. This numerical treatment is appropriate since at this stage some uncertainty exists concerning the actual values of the power levels and thermal properties.

\section{Thermal Management of the NaVigator2}

Wearable computers are highly constraint artifacts to design due to the specifie requirements such as small size, low power usage, and physical ruggedness. With such limitations, enhanced-conduction heat transfer offers an advantageous thermal design compared to those using power-consuming cooling devices, air ventilation, or bulky heat sinks. In the thermal management of the Navigator2, the overall temperature rise from the ambient environment to the heat-producing components should be kept below $20{ }^{\circ} \mathrm{C}$ since the maximum suggested temperature of most electronic devices is 
TABLE IV

Temperature Data From Heat Sink Test Bed Experiments

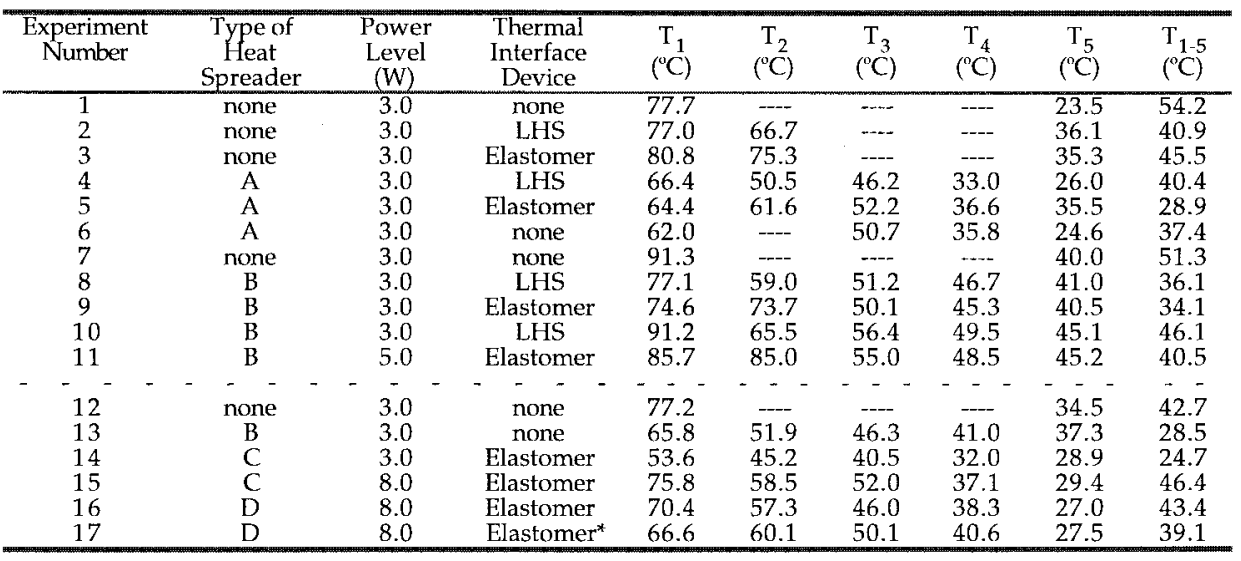

* Note: The conductive elastomer used here is $1 / 16$ in thick and has pressure sensitive adhesive on reinforced side

approximately $60{ }^{\circ} \mathrm{C}$. Furthermore, as the temperature of the electronics decreases, the reliability and life-time expectancy of the components increase exponentially [10]. Thus, the thermal design strives to achieve ways to minimize the temperature drop from the components to the outer casing where the heat is dissipated to the ambient air by natural convection.

The thermal design approach is to conduct heat from the electronic components to the outer casing by a metallic plate or heat spreader. The conductivity, shape, and thickness of the heat spreader affect the temperature drop, but the most critical point in the conduction path is the interface between the case of the electronic component and heat spreader. To minimize the contact resistance at this interface, a conductive elastomer layer consisting of silicone and aluminum oxide is used as the thermal interface to reduce air gaps between critical electronic components and the aluminum heat spreader. With this thermal management approach, the largest thermal resistance of the system becomes the convection of heat to the ambient air.

\section{A. Thermal Design Experiments for the Navigator2}

The heat sink test bed is a physical system modeling a generic wearable computer where various heat spreaders and thermal interfaces can be tested. The heat sink test bed is made of a plastic container of the approximate size of the Navigator2 [7]. Its construction allows for near air-tight conditions simulating the mostly stagnant air found inside wearable computers. Eight 7805 voltage regulators of variable power simulate the heat-producing components of a wearable computer.

In all, seventeen experiments, having power levels ranging from 3.0 to $8.0 \mathrm{~W}$, are conducted with the heat sink test bed with and without heat spreaders and with either the conductive elastomer or the LHS acting as the thermal interface. Table III summarizes the aluminum heat spreader characteristics. Because the thermocouples used in the firs eleven experiments were bulky and introduced air gaps while those used in the fina experiments are designed for surface temperature measurements, comparison should not be made between the
TABLE V

Comparative Temperature Rises in Heat Sink Test Bed Experiments

\begin{tabular}{cccc}
\hline $\begin{array}{c}\text { Experiment } \\
\text { Number }\end{array}$ & $\begin{array}{c}\text { Thermal } \\
\text { Interface } \\
\text { Device }\end{array}$ & $\begin{array}{c}\mathrm{T}_{1-3} \\
\left({ }^{\circ} \mathrm{C}\right)\end{array}$ & $\begin{array}{l}\mathrm{T}_{1-5} \\
\left({ }^{\circ} \mathrm{C}\right)\end{array}$ \\
\hline 4 & LHS & 20.2 & 40.4 \\
5 & Elastomer & 12.3 & 28.9 \\
8 & LHS & 25.9 & 36.1 \\
9 & Elastomer & 24.5 & 34.1 \\
10 & LHS & 34.1 & 46.1 \\
11 & Elastomer & 30.7 & 40.5 \\
\hline
\end{tabular}

two sets of experiments. Fig. 4 depicts the fiv locations in which the temperature data, presented in Table IV, was taken.

In the evaluation of the experimental data, the main subject of interest is the overall temperature rise $\left(T_{1-5}\right)$ between the highest heat-producing component and the stagnant air inside the test bed. Because the Epson Card has a rated maximum operating temperature of $55^{\circ} \mathrm{C}[9]$ and the stagnant air inside the Navigator 2 easily approaches $35^{\circ} \mathrm{C}$ under typical operating conditions, the overall temperature rise of the Navigator2 should be kept below $20^{\circ} \mathrm{C}$. Table V presents the temperature rises for similar cases in the firs eleven experiments to evaluate the effectiveness of the thermal interfaces. Because the conductive elastomer yields a local temperature rise consistently lower than that of the LHS by several ${ }^{\circ} \mathrm{C}$, the elastomer is chosen as the thermal interface between the heat-producing components and the heat spreader.

Three different heat spreaders are tested in addition to thermal interface materials. Doubling the heat spreader thickness from 0.0625 inches to 0.125 inches causes the overall temperature rise to fall by $6.5 \%$. Using a thinner layer of conductive elastomer with pressure sensitive adhesive reduces the contact resistance and further decreases the overall temperature rise by another $9.9 \%$. Thus, with the same exposed surface area, significan reductions in contact resistance and conductive resistance cause an overall temperature rise reduction of more than $15 \%$. Note, however, that the most efficien change in the thermal design centers around the conductive elastomer which adds little weight to the system and takes up little space. For example, the combined effect of reducing the thickness of the 


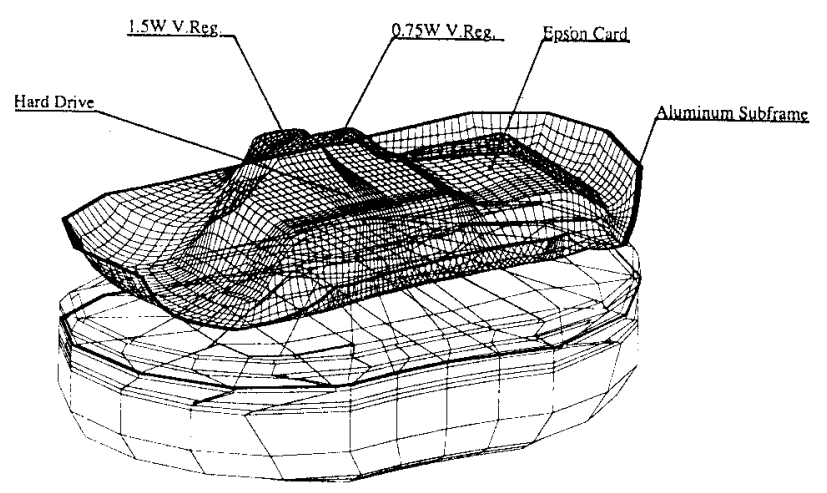

(a)

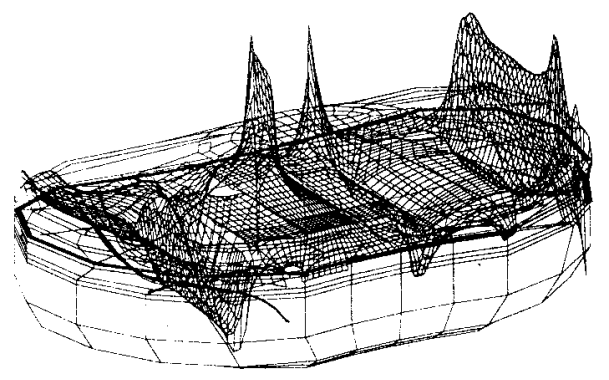

(b)

Fig. 5. Simulation results of detailed computational model showing PCB's (a) temperature profil and (b) temperature gradient.

conductive elastomer and using the adhesive backing is shown in the $32 \%$ drop of the local temperature rise $\left(T_{1-3}\right)$ from experiments 16 and 17.

In summary, the heat sink test bed experiments reveal several important findings First, although the conductive elastomer cannot boast an isothermal temperature distribution like the LHS, the conductive elastomer consistently outperforms the LHS. Second, adding surface area to the heat spreaders and using a thin conductive elastomer with pressure sensitive adhesive are two effective ways of reducing the maximum temperature of the higher heat-producing components in wearable computers.

\section{B. Numerical Simulations and Temperature Predictions}

To predict the temperature distribution within the Navigator2, numerical simulations are performed with a spectral element code using two computational models. One computational model is designed with simplifie geometry, grouping various components and estimating their material properties, while the other is constructed in great geometric detail. Material properties of the simplifie and detailed computational models are presented in [7]. The Navigator2 has a complex, rounded shape, as shown in Fig. 1; whereas, the simplifie model is rectangular and has the same approximate surface area and volume as the Navigator2. In the mesh-generation process, the Navigator 2 is represented by a series of layers built up on top of one another. Each layer is composed of blocks representing different components and segments. Fig. 5(a) and (b) presents the detailed computational model of the Navigator2.
TABLE VI

Numerical Simulations of Simplified Model of NaVigator2

\begin{tabular}{lllll}
$\begin{array}{l}\text { Mesh } \\
\text { Type }\end{array}$ & $\begin{array}{l}\text { Exposed } \\
\text { Heat } \\
\text { Spreader } \\
\left.\text { Area (in }{ }^{2}\right)\end{array}$ & $\begin{array}{c}\mathrm{k}_{\text {aluminum }} \\
(\mathrm{W} / \mathrm{m} \mathrm{K})\end{array}$ & $\begin{array}{l}\mathrm{k}_{\text {elastomer }} \\
(\mathrm{W} / \mathrm{m} \mathrm{K})\end{array}$ & $\begin{array}{l}\mathrm{T}_{\max } \\
\left({ }^{(} \mathrm{C}\right)\end{array}$ \\
\hline $\mathrm{A}$ & 74 & 85 & 0.50 & \\
$\mathrm{~B}$ & 74 & 170 & 0.50 & 54.8 \\
$\mathrm{C}$ & 124 & 170 & 0.50 & 46.7 \\
$\mathrm{D}$ & 149 & 85 & 0.50 & 46.2 \\
$\mathrm{E}$ & 124 & 170 & 1.75 & 45.5 \\
$\mathrm{~F}$ & 173 & 85 & 0.50 & 44.4 \\
$\mathrm{G}$ & 149 & 170 & 0.50 & 44.2 \\
$\mathrm{H}$ & 198 & 85 & 0.50 & 43.1 \\
$\mathrm{I}$ & 173 & 170 & 0.50 & 42.5 \\
$\mathrm{~J}$ & 198 & 170 & 0.50 & 43.1 \\
\hline
\end{tabular}

A lumped analysis is undertaken to estimate the contact resistance of the conductive elastomer. To approximate the effect of the contact resistance, the conductivity of the elastomer is firs reduced by approximately $2 / 3$ to effectively model air gaps at the two interfaces of the conductive elastomer with both the heat spreader and the electronic components. A more detailed analysis shows that conduction of heat from the electronics to the heat spreader is roughly twice as efficien when using the conductive elastomer, compared to no interface at all.

Table VI shows that the maximum temperatures predicted in the simulations of the simplifie model are a function of the heat spreader area exposed to the air, the conductivity of the heat spreader and subframe, $k_{\text {aluminum, }}$, and the conductivity used for the conductive elastomer, $k_{\text {elastomer }}$. The effect of enlarging the surface area of the heat spreader is indirectly accounted for by increasing the convection coefficien used for that area since the convective thermal resistance is inversely proportional to the convection coefficien and the exposed heat spreader area. Even though this method of simulating larger exposed heat transfer areas does not take into account the added thermal resistance due to a longer conduction path in the heat spreader, the resulting overall temperature rise is only slightly less than what would actually occur because of the relatively high conductivity of the metal heat spreaders.

From simulations (A) through (E) in Table VI, it becomes apparent that regardless of the conductivity used for the conductive elastomer or metal, the temperature begins to fall in significan amounts only when the heat spreader surface area exposed to air is enlarged. Simulations (F) through (J) indicate that satisfactory temperature levels are achieved as long as the exposed surface area exceeds $125 \mathrm{in}^{2}$. Therefore, the thermal design of the Navigator 2 calls for internal enhancedconduction/external natural-convection heat transfer scheme employing 1/16 inch-thick conductive elastomer with pressure sensitive adhesive as the thermal interface and $150 \mathrm{in}^{2}$ of exposed surface area of the $1 / 8$ inch-thick aluminum heat spreader.

The simulations of the detailed model do not precisely coincide with those from the simplifie model. For example, using the same material properties and convective thermal resistance from the heat spreader of the simplifie model, the simulation of the detailed computational model predicts a maximum temperature rise that is $23 \%$ less than that of case B in Table VI. This discrepancy is due to three reasons: the absence of the hard drive, the geometric approximations of 


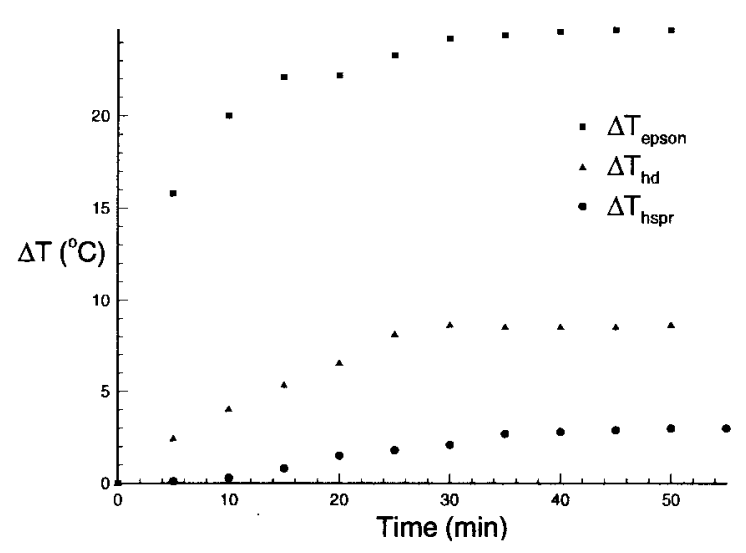

Fig. 6. Temperature data from open-air experiments.

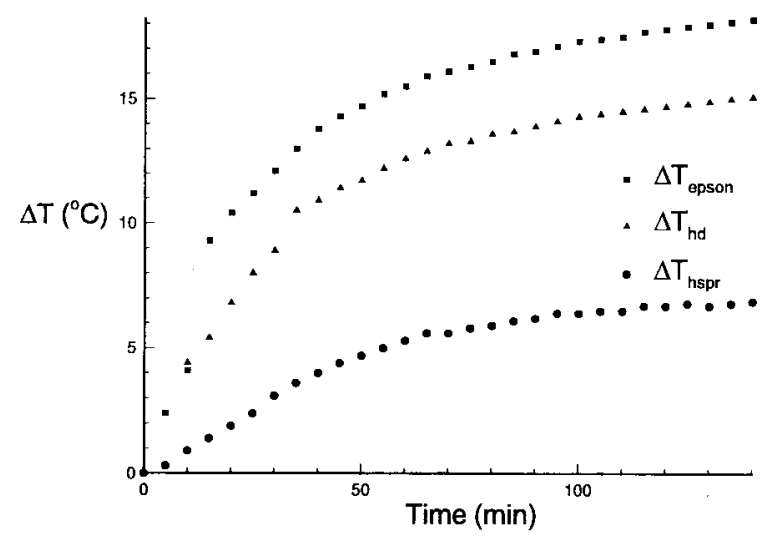

Fig. 7. Temperature data from closed-housing experiments without protective harness.

the simplifie model, and the difference between modeling the air inside the housing as stagnant air in the simplifie model and modeling the air with conservative convective boundary conditions in the detailed model.

The temperature profil and temperature gradients along the length of the printed circuit board are depicted in Fig. 5(a) and (b), respectively. Although the Epson Card produces the most heat approximated at $4.5 \mathrm{~W}$, the $1.5 \mathrm{~W}$ voltage regulator is the hottest component due to its small size giving it a volumetric heat generation that is more dense than that of the Epson Card by a factor of 2.5. Furthermore, the hard drive, being close to the voltage regulators and isolated from the heat spreader, causes its temperature to exceed that of the Epson Card even though its volumetric heat-generation density is only 8\% that of the Epson Card. Fig. 5(b) shows that the highest temperature gradients, and thus zones of thermally-induced stresses, occur in the printed circuit board near the edges of the Epson Card and voltage regulators. These simulations clearly show the critical roles played by the size and location of components in the thermal design.

\section{Temperature Measurements of the Navigator2}

Three physical experiments are performed on the actual wearable computer prototype to demonstrate the effectiveness of the fina thermal design of the Navigator2: an open-

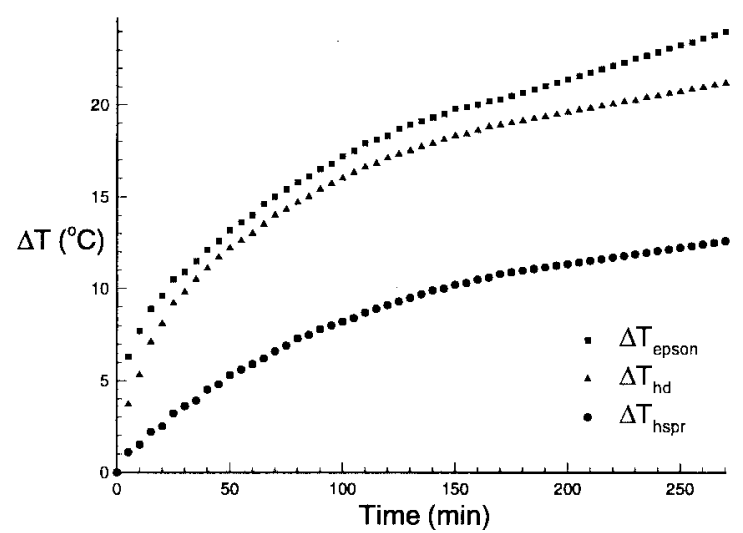

Fig. 8. Temperature data from closed-housing experiments with protective harness.

air experiment, a closed-housing experiment, and a closedhousing with protective harness experiment. The results shown in Figs. 6-8 are the time-dependent temperature rises from the ambient air to the component of interest. In the open-air test, the heat spreader underneath the PCB is detached from the Epson Card such that the electronics are exposed to the open air with only the voltage regulators in thermal contact with the aluminum subframe. In the second experiment, the housing of the Navigator2 is closed, there is no ventilation to the ambient air, and the PCB board, heat spreader, and aluminum subframe are in thermal contact with each other. Comparing Figs. 6 and 7 shows that the thermal design with the heat spreader and aluminum subframe, acting as the heat sink, allows the metal to absorb much of the transient thermal energy, taking three times as long to reach steady-state when the heat spreader is attached to the aluminum subframe. At the end of $50 \mathrm{~min}$, the open-air results show the Epson Card reaching the steadystate temperature rise of $24.0{ }^{\circ} \mathrm{C}$ which is $69 \%$ higher than its temperature rise in the closed-housing test. Furthermore, at steady-state, the closed-housing test shows the temperature rise of the Epson Card is reduced by $24 \%$ from that of the steadystate value in the open-air test. Therefore, the conduction heat transfer path using the conductive elastomer, the heat spreader, and the aluminum subframe constitutes an effective thermal design that is simple to implement, rugged, and requires no power for operation.

Since the Navigator2 is worn around the waist with a protective harness as shown in Fig. 1, the third test is conducted to ascertain the effectiveness of the thermal design when enclosed in the harness. The protective harness consists of thermally insulative materials that surround the housing and the aluminum subframe. Fig. 8 shows that steady-state conditions are not reached after $4.5 \mathrm{~h}$, indicating that the harness absorbs thermal energy as well as reduces convection from the subframe. Comparison of the results to the closedhousing test, after reaching steady state in approximately $3 \mathrm{~h}$, indicates that the temperature rise of the Epson Card with the harness is only increased by $11 \%$. Therefore, for moderate usage or lower power levels achieved by efficien power management, the harness does not constitute a hazard to the electronics due to the time lag and heat sink effect of the 
thermal design. However, for continuous use of the Navigator2 of more than $4 \mathrm{~h}$, the insulative behavior of the harness begins to negate the advantages of the thermal design and overtakes the expected temperatures of the open-air test.

\section{CONCLUSION}

The process of designing a wearable computer involves different disciplines working in parallel with design dependencies that intersect at critical stages where design decisions need to be made. In practice, the concurrent design of a wearable computer requires interdisciplinary communication throughout the design evolution. The design of the Navigator2 illustrates the application of concurrent engineering to produce a dual architecture wearable computer with a 486-level processor and a speech recognition digital signal processor. This novel architecture results in a factor of two reduction in power consumption.

The power reduction at the hardware level along with the power management at the software level enables a thermal design solution employing enhanced-conduction heat transfer. This thermal design employs a thin conductive elastomer with pressure sensitive adhesive as the thermal interface, $150 \mathrm{in}^{2}$ exposed surface area of the 1/8 inch-thick aluminum heat spreader, and an aluminum subframe acting as both heat sink and structural support.

In concurrent thermal design, the thermal designer works closely with industrial, electronic and software designers, adjusting thermal design specification to meet the overall design goals. A framework of concurrent thermal engineering consisting of three basic stages helps to maintain interdisciplinary interaction while satisfying thermal design goals. The firs stage of the thermal design selects and evaluates the required cooling arrangement. The second stage investigates the thermal contact between the aluminum heat spreader and the electronics where physical experimentation becomes the primary approach for selection and implementation of appropriate thermal contact devices. The third stage consists of numerical simulations with both simplifie and detailed computational models. Simplifie models enable fast parametric studies and sensitivity analysis, whereas detailed models ascertain the effectiveness of the thermal design and predict accurately the temperature distribution of the Navigator2 before it is manufactured.

The thermal design of the Navigator2 is a practical example of concurrent thermal engineering. Because industrial design specification require the wearable computer to be ventless, small, lightweight and rugged while the electrical design requires a power level on the order of 10 Watts, it is decided early in the thermal design to explore enhancedconduction heat transfer with aluminum heat spreaders to transfer the heat to the outside housing to be dissipated by natural convection. In this design, the most critical point is the contact resistance between the electronics and heat spreader. With a simple experimental apparatus, two appropriate thermal interfaces are investigated: a liquid heat sink (LHS) and a conductive elastomer. The LHS is a plastic pouch fille with fluoriner liquid that produces an isothermal platform, while the conductive elastomer is made of silicone with aluminum oxide fille that conforms to the surface of the electronic components. In experiments conducted on these devices, the elastomer consistently yields a smaller temperature rise from the electronics to the surrounding air and is, therefore, chosen as the thermal interface device. The numerical simulation of the simplifie model of the Navigator2 enables the thermal designer to assess the adequacy of the exposed heat spreader surface area and the performance of the conductive elastomer. Although the detailed model is not needed for design purposes, it verifie the level of accuracy of the simplifie model while providing a more accurate rendition of the Navigator 2 thermal performance. Temperature profile and temperature gradients allow the thermal designer to quickly ascertain the location of hot spots and thermal stress concentrations.

Field evaluation of the Navigator2 wearable computer, used for aircraft inspection at McClellan Air Force Base, indicates not only $20 \%$ savings in inspection time but also a dramatic reduction of inspection data entry time from hours to minutes.

\section{REFERENCES}

[1] C. H. Amon, J. S. Nigen, D. P. Siewiorek, A. Smailagic, and J. M. Stivoric, "Concurrent design and analysis of the navigator wearable computer system: The thermal perspective," IEEE Trans. Comp., Packag., Manufact. Technol., vol. 18, pp. 567-577, 1995.

[2] C. H. Amon, S. Finger, D. P. Siewiorek, and A. Smailagic, "Integration of design education, research and practice at Carnegie Mellon university: A multi-disciplinary course in wearable computer design," in Proc. ASEE/IEEE 25th Annual Frontiers in Education Conf., Atlanta, GA, 1995, pp. 14-22.

[3] A. Bar-Cohen and A. D. Kraus, Advances in Thermal Modeling of Electronic Components and Systems, vol. 3, New York: Hemisphere, 1988.

[4] M. Culbert, "Low power hardware for a high performance PDA," in Proc. IEEE COMPCON '94, San Francisco, CA, 1994, pp. 144-147.

[5] R. Dobkin, C. Nelson, and S. Pietkiewicz, "Power conditioning for notebook and palmtop systems," in Proc. Analog and Mixed-Signal Design Conf. Proceedings, Burlingame, CA, 1992.

[6] E. Egan and C. H. Amon, "Cooling strategies for embedded electronic components of wearable computers fabricated by shape deposition manufacturing," in Proc. IEEE InterSociety Conf. Thermal Phenomena, Orlando, FL, 1996, pp. 13-20.

[7] _ , "Thermal design of wearable computers: Application to the navigator2, thermal management devices, and embedded electronics," Carnegie Mellon University, Pittsburgh, PA, EDRC Rep. 24-123-95, 1995.

[8] S. Finger, J. Stivoric, C. H. Amon, L. Gursoz, F. Prinz, D. Siewiorek, A. Smailagic, and L. Weiss, "Reflection on a concurrent design methodology: A case study in wearable computer design," ComputerAided Design, Special Issue on Concurrent Design, vol. 28, no. 5, pp. 393-404, 1996.

[9] J. Hanna, personal reference, Epson America, Inc., NJ, 1995.

[10] R. D. Johnson, "Efficien cooling increases component and equipment reliability," Electrotechnol., vol. 15, Reprinted by Technical Manager, IREE Monitor, Redpoint Ltd. U.K, 1989.

[11] T. Lee, B. Chambers, and M. Mahalingam, "Application of CFD technology to electronic thermal management," IEEE 5503/94, pp. 411-420, 1994

[12] J. S. Nigen and C. H. Amon, "Concurrent thermal designs of PCB's: Balancing accuracy with time constraints," IEEE Trans. Comp., Hybrids, Manufact. Technol., vol. 15, pp. 850-859, 1992.

[13] S. B. Sathe and B. G. Sammakia, "Natural-convection in an open ended horizontally heated cavity: A numerical study with applications to laptop computers," in Proc. ASME National Heat Transfer Conf., 1995, vol. 1, pp. 81-93.

[14] A. Smailagic and D. P. Siewiorek, "A case study in embedded systems design: The VuMan 2 wearable computer," IEEE Design and Test of Computers, vol. 10, pp. 56-67, 1993.

[15] _ "Interacting with CMU mobile computers," IEEE Personal Communication, vol. 3, pp. 6-16, 1996 
[16] D. P. Siewiorek, A. Smailagic, J. C. Y. Lee, and A. R. A. Tabatabai, "Interdisciplinary concurrent design methodology as applied to the navigator wearable computer system," J. Comput. Software Eng., vol. 2, no. 3, pp. 259-292, 1994.

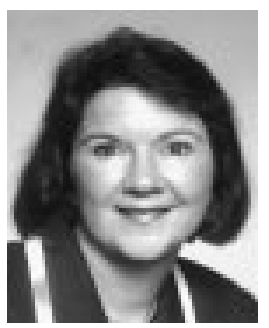

Cristina H. Amon (SM'95) received the B.S degree in mechanical engineering from the University of Simon Bolivar, Venezuela, and the M.S. and Sc.D. degrees in mechanical engineering from the Massachusetts Institute of Technology, Cambridge, in 1985 and 1988, respectively.

She is a Professor in the Mechanical Engineering Department at Carnegie Mellon University. She serves as Associate Editor of the IEEE Transactions on Components, Packaging, and Manufacturing TeChnology, Associate Editor of the Electronic Packaging G\&B Book Series, and Co-Editor of ASME Heat Transfer Division publications. Her research interests include computational flui dynamics and heat transfer, thermal management of electronic portable and wearable computers, electronic packaging, concurrent thermal design, thermal phenomena in manufacturing processes, and heat transfer augmentation techniques.

Dr. Amon received the NSF Initiation Award in 1989, the G.T. Ladd Award for Excellence in Research in 1991, the SAE Teetor Educational Award in 1994, the ASEE NCS Outstanding Teaching Award, the Pi Tau Sigma Honorary Member in 1995, and the ASEE Outstanding Campus Rep Award in 1996. She is a member of ASME, AIAA, ASEE, SWE, Pi Tau Sigma, and Sigma Xi. She is Chair of the ASME HTD Committee on Honors Nominations and serves on the ASME Technical Committees on Heat Transfer in Electronic Equipment, Fundamentals of Heat Transfer and Computational Heat Transfer.

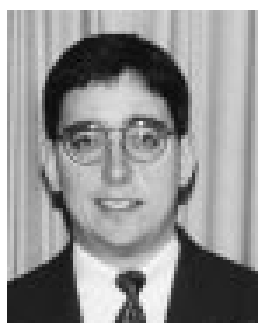

Eric R. Egan received the B.S. degree in mechanical engineering from Pennsylvania State University, University Park, in 1994, and the M.S. degree in mechanical engineering from Carnegie Mellon University, Pittsburgh, PA, in 1996.

His research interests include the use of numerical simulations and physical experimentation in the thermal design of portable, wearable computers, and the enhancement of thermal conductivity of polymer composites for embedded electronic applications.

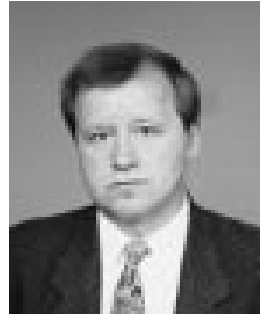

Asim Smailagic (A'93) received the Ph.D. degree in computer science from the University of Sarajevo in 1984 .

$\mathrm{He}$ is a Faculty Member at Carnegie Mellon University's Engineering Design Research Center, Pittsburgh, PA. He was a Professor of computer science and computer engineering at the University of Sarajevo, and a Visiting Professor at the University of Leeds, U.K. He participated in several major projects that represent milestones in the evolution of computer system architectures: Carnegie Mellon's Cm* multiprocessor system, the Edinburgh Multi-Microprocessor Assembly (EMMA), and the current Carnegie Mellon projects on the wearable multicomputer and smart modules dedicated computers. He served on numerous advisory boards and review panels, as well as on the research journal editorial boards, such as the Journal of Computing and Information Technology. His research interests include hardware-software co-design, wearable computers and their applications, rapid prototyping, and audio-visual interfaces to computer systems.

Dr. Smailagic received a research fellowship from the University of Edinburgh and a Fulbright Postdoctoral Award at Carnegie Mellon. He was an invited Program Chairman of the Cambridge Colloquium on Massively Parallel Computing, and is a member of ACM.

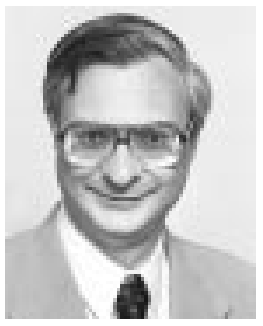

Daniel P. Siewiorek (S'67-M'72-SM'79-F'81) received the B.S. degree in electrical engineering from the University of Michigan, Ann Arbor, in 1968, and the M.S. and Ph.D. degrees in electrical engineering (minor in computer science) from Stanford University, Stanford, CA, in 1969 and 1972, respectively.

The Buhl Professor of Electrical and Computer Engineering and Computer Science at Carnegie Mellon University, Pittsburgh, PA, he helped to initiate and guide the $\mathrm{Cm} *$ project that culminated in an operational 50-processor multiprocessor system. He has designed or been involved with the design of nine multiprocessor systems and has been a key contributor to the dependability design of over two dozen commercial computing systems. He leads an interdisciplinary team that has designed and constructed eight generations of mobile computing systems. He has served as a consultant to several commercial and government organizations, while serving on six technology advisory committees. He has written eight textbooks in the areas of parallel processing, computer architecture, reliable computing, and design automation, in addition to over 300 papers. He has served as Associate Editor of the Computer System Department of the Communications of the Association for Computing Machinery. He is currently Director of the CMU Engineering Design Research Center.

Dr. Siewiorek received the Frederick Emmons Terman Award from the American Society for Engineering Education in 1983 for outstanding young electrical engineering educator and received the IEEE Computer Society and Association for Computing Machinery (ACM) Eckert-Mauchly Award in 1988 for his outstanding contributions in parallel computer architecture, reliability, and computer architecture education. He is a member of the 1994 inaugural class of ACM Fellows and a past Chairman of the IEEE Technical Committee on Fault-Tolerant Computing. He is a member of ACM, Tau Beta $\mathrm{Pi}$, Eta Kappa $\mathrm{Nu}$, and Sigma Xi. 